\section{AOP-Nachsorge ist extrabudgetär}

— Die Präambel zum Kapitel 31.4 (Postoperative Behandlungskomplexe) legt fest, dass die Gebührenordnungspositionen dieses Abschnittes vom Operateur oder auf Überweisung vom weiterbehandelnden Vertragsarzt nach ambulanter Durchführung eines Eingriffs des Abschnittes 31.2 ("Ambulante Operationen“) berechnet werden können. Da diese Leistungen extrabudgetär vergütet werden, sollte man die Rahmenbedingungen beachten.

Werden die Gebührenordnungspositionen des Abschnitts 31.4 abrechnet, muss aus dem Abrechnungsschein das Datum des zugrunde liegenden operativen Eingriffes ersichtlich sein. Für Vertragsärzte des hausärztlichen Versorgungsbereichs gibt es zur Abrechnung nur die Einheitsnummer 31600 EBM für alle Eingriffe. In dem Zeitraum vom 1. bis zum 21. postoperativen Tag kann aber nur einmalig dieser Behandlungskomplex des Abschnittes 31.4 abgerechnet werden.

\section{MMW Kommentar}

Das OP-Datum sollte man deshalb nicht vergessen, da ansonsten die Leistung nicht vergütet werden kann. Die Einschränkung, dass der postoperative Behandlungskomplex erst mit dem zweiten Arzt-PatientenKontakt ab dem 1. Tag nach der Operation abgerechnet werden kann, gilt aufgrund der Präambel zum Kapitel 31.2.1 Nummer 5 nur bei Erbringung der postoperativen Behandlungskomplexe durch den Operateur selbst. Wichtig ist die chronologisch korrekte Eintragung, da man sich z. B. mit einem einfachen Zahlendreher schnell außerhalb der 21 Tage befinden oder bei mehreren Operationen innerhalb von 21 Tagen mehr als einen Nachsorgekomplex eintragen kann. Formal spricht der EBM zwar von einer Überweisung des Operateurs, eine entsprechende Aufforderung im Arztbrief des Operateurs ist aber auch ausreichend und wird bei der Abrechnung anerkannt.

\section{Nachträgliche Rezeptänderung}

— Wenn ein Arzt bei der Ausstellung eines Rezeptes eine nachträgliche Änderung handschriftlich vornimmt, muss diese abgezeichnet und ein Datum eingefügt werden. Nur bestimmte Formfehler auf einem Rezept können von der Apotheke korrigiert oder ergänzt werden.

\section{MMW Kommentar}

Dies regelt §11 Absatz 1 der Arzneimittelrichtlinie des Gemeinsamen Bundesausschusses (G-BA), wonach die Versorgung mit Arzneimitteln im Rahmen der vertragsärztlichen Versorgung eine Verordnung der behandelnden Ärztin oder des behandelnden Arztes auf einem ordnungsgemäß ausgestellten Kassenrezept (Vordruck Muster 16 gem. §87 Abs. 1 SGB V) voraussetzt. Änderungen und Ergänzungen zu einer ausgestellten Verordnung bedürfen der erneuten Unterschrift des behandelnden Arztes mit Datumsangabe.

\title{
Wie kann ich eingeschränkt verordnungsfähige Arzneimittel kennzeichnen?
}

\begin{abstract}
- Seit dem 1. Juli 2012 sind neue Vorschriften für die ärztliche Arzneiverordnungssoftware (AVS) in Kraft, die von der KBV zusammen mit dem Spitzenverband der Gesetzlichen Krankenkassen (SpiK) beschlossen wurden.

Auf Rezepten dürfen danach nur noch Produkt- bzw. Wirkstoffbezeichnung, Wirkstärke, Darreichungsform und Normgröße angegeben werden. Weitere Angaben haben gesetzlichen und rahmenvertraglichen $\mathrm{Be}$ stimmungen zu entsprechen. Darüber hinaus gehende Angaben auf dem Rezept (z. B. , "PZN “, , ,keine Substitution“, „,kein Aut idem", "das Medikament ist zuzahlungsbe-
\end{abstract}

freit" oder Verordnungsbegründungen) sind nicht mehr zulässig.

\section{MMW Kommentar}

Da die Verordnung von einigen apothekenpflichtigen Arzneimitteln nur bei bestimmten Erkrankungen eine Kassenleistung darstellt, wie beispielsweise die Eisensubstitution bei der Diagnose Eisenmangelanämie oder die Vitamin-B12-Gabe bei der perniziösen Anämie, stellt sich die Frage, wie bei einer Prüfung der Einzelverordnung erkannt werden kann, dass die Diagnose beim Patienten vorlag? Hier ist zu beachten, dass bei einer eventuellen Wirtschaftlichkeitsprüfung (Einzel- fallprüfung) immer Verordnungsblatt und Abrechnungsschein des Patienten zusammengeführt werden. Zudem ist auch die Dokumentation in der Patientenkarteikarte geeignet, die Verordnung zu begründen. Anders ist die Situation allerdings bei der Hilfsmittelverordnung. Die Hilfsmittel-Richtlinien verpflichten den Arzt, eine Diagnose auf dem Arzneiverordnungsblatt (Muster 16) bei der Hilfsmittelverordnung anzugeben. Das bedeutet, bei der Arzneimittelverordnung darf keine Diagnose auf das Rezept, bei der Hilfsmittelverordnung muss eine Diagnose auf das Rezept. 